\title{
Atlantide e altri pays chimériques: mito platonico e poesia nelle Grazie di Foscolo e in alcuni autori europei coevi
}

Atlantide et autres pays chimériques : mythe platonicien et poésie dans Le Grazie de Foscolo et chez d'autres auteurs européens contemporains Atlantis and Other pays chimériques: Platonical Myth and Poetry in Foscolo's Grazie and in Other Contemporary European Authors

\section{Chiara Lombardi}

\section{OpenEdition}

\section{Journals}

Edizione digitale

URL: http://journals.openedition.org/cei/2380

DOI: $10.4000 /$ cei. 2380

ISSN: 2260-779X

Editore

UGA Éditions/Université Grenoble Alpes

Edizione cartacea

Data di pubblicazione: 30 juin 2015

Paginazione: 85-100

ISBN: 978-2-84310-289-9

ISSN: $1770-9571$

\section{Notizia bibliografica digitale}

Chiara Lombardi, «Atlantide e altri pays chimériques: mito platonico e poesia nelle Grazie di Foscolo e in alcuni autori europei coevi», Cahiers d'études italiennes [Online], 20 | 2015, online dal 01 janvier 2017, consultato il 26 mars 2021. URL: http://journals.openedition.org/cei/2380 ; DOI: https://doi.org/ $10.4000 /$ cei. 2380 


\title{
ATLANTIDE E ALTRI PAYS CHIMÉRIQUES: MITO PLATONICO E POESIA NELLE GRAZIE DI FOSCOLO E IN ALCUNI AUTORI EUROPEI COEVI
}

\author{
Chiara Lombardi \\ Università di Torino
}

Il paesaggio delle Grazie - composto sull'intreccio armonioso tra narrazione storica, pittura poetica e morale allegorica — si sviluppa tra la Grecia «antichissima» dell'inno I a Venere, l'Italia del tempo di Foscolo, rivolto allo «stato possibile futuro dell' incivilimento maggiore», nell'inno II dedicato a Vesta, e l'Atlantide dell'inno III, dedicato a Pallade, "più metafisico perché allude più di proposito al potere degli affetti, e dell'arti umane su la forza de le umane passioni; e ci trasporta in un paese ideale» ${ }^{ }$.

Il mito di Atlantide, presentato da Platone nel prologo del Timeo e nel Crizia ${ }^{2}$, ricompare però nella riscrittura foscoliana privato dell'originaria simbologia che indicava l'isola come spazio della dismisura e della degenerazione morale e politica, in contrapposizione con la sobrietà e il buon governo dell'antica Atene ${ }^{3}$. Anche la punizione degli dèi, con cui si chiudeva il Crizia, è un elemento su cui gioca il recupero dell'immaginario

I. U. Foscolo, Appunti sulla ragion poetica del Carme (EN I, p. 973). Dove non riferite all'Edizione Nazionale, le citazioni dei versi sull'Atlantide dell'inno III delle Grazie sono tratte dall'edizione di M. Puppo (in U. Foscolo, Opere, a cura di M. Puppo, Milano, Mursia, 1966, pp. 38-75) dove è proposta una versione completa del testo (basata sulla ricognizione di Chiarini). Per gli aspetti interpretativi, terrò anche conto dell'edizione a cura di S. Orlando, U. Foscolo, Le Grazie. Carme ad Antonio Canova, Brescia, Paideia, 1974. Cfr. Id., Il mito di Atlantide nelle "Grazie» del Foscolo, «Italianistica», III, I974, pp. 33-53.

2. L'edizione di riferimento per le citazioni platoniche è Platon, Euvres complètes, Paris, «Les Belles Lettres»; per questi testi, in particolare: Timée-Critias, texte établi et traduit par A. Rivaud, Paris, "Les Belles Lettres», 1949. Le traduzioni dal greco e dall'inglese, salvo specificazione, sono personali.

3. Secondo i più recenti studi, il mito va letto in coerenza con le teorie del filosofo sulla complementarietà tra mythos e logos, e in relazione agli altri miti politici elaborati nella Repubblica, nel Politico, nelle Leggi (R. Weil, L'«archéologie» de Platon, Paris, PUF, 1959; G. Arrighetti, Platone tra mito, poesia e storia, "Studi Classici e Orientali», XLI, I99I, pp. 13-34; P. Vidal-Nacquet, Atlantide. Breve storia di un mito, Torino, Einaudi, 2006). Dal punto di vista politico, risulta particolarmente convincente l'interpretazione, di recente ripresa da Vidal-Naquet nello studio sopra citato, che vede Atlantide come figura pantografata dell'Atene periclea in 
antico: se in Platone gli dèi colpiscono la hybris degli abitanti di Atlantide, coinvolgendo nella guerra e nella distruzione anche l'antica Atene per cancellare una memoria che si deve rinnovare nel presente (Ti., 25a-26a; Criti., I2Ia-c), nelle Grazie la punizione sancisce l'allontanamento degli uomini non dall'ordine politico, ma dal culto per le arti. Nell'inno III dell'opera, infatti, l'isola è rappresentata come «aurea terra» cinta di cielo "pervio soltanto ai numi» (III, vv. 98-IOo), dopo che i suoi abitanti ne furono espulsi e scacciati dentro l'Asia per l'ira di Minerva, poiché «il ricco suolo e gl'imenei lascivi / fean pigri all'arti e sconoscenti a Giove» (III, vv. 97-98). All'«alta / reggia» (III, vv. 56-57) di Atlantide, tuttavia, la dea ferma la sua quadriga per offrire questo spazio di cultura sublime e di felicità incorrotta come «dono / alle timide Grazie» (III, vv. I2I-I22), le cui «opere» di bellezza dipingono quel paese ideale prima di riconsegnarlo ai poggi fiorentini del tempo presente 4 . All'Atene del passato, cancellata dalla storia, si sostituisce quindi Atlantide, che l'«errante Fantasia» (III, v. 28) ravviva e conserva intatta.

Tra Settecento e Ottocento, in generale, il mito di Atlantide torna a figurare nei testi letterari proprio a partire dalla confutazione del sistema metafisico platonico (o, meglio, dall'acquisizione e dal rovesciamento del platonismo): come parodia (nel poema eroicomico di Casti Gli animali parlanti o nei Paralipomeni leopardiani, ad esempio) ${ }^{5}$ o come nostalgia del compiuto, ricerca del possibile, miraggio o pays chimérique 6 . Anche la definizione foscoliana, sopra riportata, dell'inno come piu metafisico implica un punto di vista che ridefinisce, su basi radicalmente diverse, la concezione platonica di trascendenza riportandola dal piano dell'idea a quella dell' ideale, dall'ambito teoretico a quello estetico ed etico.

In questo contributo prenderò quindi in considerazione l'immagine di Atlantide nel III inno delle Grazie, alla luce delle trasformazioni dell'idealismo platonico e del mito nella poesia europea coeva a Foscolo ${ }^{7}$.

lotta contro Sparta, tendente all'eccesso e alla prevaricazione, alla degenerazione rispetto alle sue origini divine, quindi in contrapposizione con l'immagine (simbolica) dell'antichissima, sobria e ben regolata, Atene.

4. M. Cerruti, Foscolo, gli antichi segni di luce, in L'«inquieta brama dell'ottimo», Palermo, Flaccovio, I982, pp. I75-I9I.

5. Per questo percorso, mi permetto di rimandare a: C. Lombardi, "La sacra isola sotto il sole». Il mito di Atlantide in Platone, Casti, Foscolo, Leopardi, Civitavecchia, Prospettiva editrice, 2006.

6. B. Backzo, Lumières de l'utopie (I978), tr. it. L'utopia. Immaginazione sociale e rappresentazioni utopiche nell'età dell'illuminismo, Torino, Einaudi, 1979; cfr. M. Cerruti, La ragione felice e altri miti del Settecento, Firenze, Olschki, I973; Id., La guerra e i Lumi nel Settecento italiano, Torino, Thélème, 2000.

7. J. J. Chambliss, Imagination and Reason in Plato, Aristotle, Vico, Rousseau and Keats: An Essay on the Philosophy of Experience, Dordrecht, Springer, 1974; T. Kennedy, Platonism in Keats' "Ode on a Grecian Urn", "Philological Quarterly», LXXV, 1996, n I, pp. 85-I07; L. Cooper, Wordsworth's Knowledge of Plato, "Modern Language Notes", XXXIII, I9I8, nº 8, pp. 497-499; R. Dell'Erede, La triade o l'unità: mitologie foscoliane e 
Come cercherò di dimostrare, l'opera si presta a un fruttuoso dialogo intertestuale con il romanticismo europeo ${ }^{8}$, con autori quali Leopardi, Coleridge, Wordsworth, Keats e Hölderlin, soprattutto per il farsi di concetti teorici come l'immaginazione, la bellezza, l'allegoria9 .

\section{Il platonismo nella poesia europea e in Foscolo: immaginazione, bellezza, allegoria}

Nel pensiero di Vico e di Rousseau e nella poesia europea della prima metà dell'Ottocento si individuano significative forme di acquisizione e metamorfosi del pensiero platonico ${ }^{10}$. Da una parte materialismo e sensismo negano rispettivamente alla metafisica sostanza ontologica e valore conoscitivo, dall'altra l'idealismo tende a ricondurre l'essere al soggetto, all'attività del pensiero e della produzione artistica; la nozione di idea platonica tende dunque ad essere ripresa e concepita come ideale che alimenta l'energia e la forza desiderante del soggetto e si trasforma in poesia, ricreando il mondo, reale e trascendente, nelle forme dell'immaginazione.

Questa trasformazione è molto evidente nella riflessione di Leopardi. Nello Zibaldone, commentando l'Essai sur le goût di Montesquieu, il poeta afferma l'impossibilità di sostenere un'idea aprioristica del bello per la quale possa essere formulato un giudizio oggettivo. «Il tipo o la forma del bello non esiste, e non è altro che l'idea della convenienza», osserva il poeta ${ }^{\text {II }}$. Con l'uso dei termini «tipo» e "forma»" ${ }^{\mathrm{I2}}$, Leopardi si riferisce al sistema

suggestioni neoplatoniche, "Mario \& Mario. Annuario di critica letteraria italiana e comparata», I995, pp. 63-89; R. Gilodi, Tradurre Platone, in Id., Origini della critica letteraria. Herder, Moritz, Fr. Schlegel e Schleiermacher, Milano, Mimesis, 2013, pp. IO3-II9.

8. Foscolo viaggia in Francia a partire dal 1804 e in Inghilterra dal I8I6 (cfr. J. Lindon, Studi sul Foscolo inglese, Pisa, Giardini, 1987; S. Parmegiani, Ugo Foscolo and English Culture, London, Legenda, 20II).

9. Per un confronto tra il neoclassicismo foscoliano e il romanticismo europeo si considerino M. Praz, Foscolo tra romanticismo e neoclassicismo, in "Cultura e scuola», LXVII, 1978, pp. 17- 29, e L. Derla, L'isola, il velo, l'ara. Allegoria e mito nella poesia di Ugo Foscolo, Genova, Edizioni Culturali Internazionali, 1984. Si vedano inoltre: K. Kroeber, The Artifice of Reality. Poetic Style in Wordsworth, Foscolo, Keats, and Leopardi, Milwaukee, University of Wisconsin Press, 1964; T. Klinkert, Literarische Selbstreflexion im Medium der Liebe: Untersuchungen zur Liebessemantik bei Rousseau und in der europäischen Romantik (Hölderlin, Foscolo, Madame de Staël und Leopardi), Freiburg, Rombach, 2002; C. Del Vento, Un allievo della Rivoluzione. Ugo Foscolo dal «noviziato letterario" al "nuovo classicismo» (1795-I806), Bologna, Clueb, 2003; E. Neppi, Foscolo, pensatore europeo. La dualità dell'essere nell' "Orazione pavese», in U. Foscolo, Dell'origine e dell'ufficio della Letteratura, a cura di E. Neppi, Firenze, Olschki, 2005, pp. 5-90; G. Cordibella, Hölderlin in Italia. La ricezione letteraria, Bologna, il Mulino, 2009.

IO. Si pensi anche al romanzo epistolare: V. Cuoco, Platone in Italia, a cura di A. De Francesco e A. Andreoni, Bari, Laterza, 2006.

II. G. Leopardi, Zibaldone, a cura di R. Damiani, Milano, Mondadori, 1997, f. I54, p. I84.

I2. Damiani osserva che nell'autografo è cancellato bello ideale (ivi, n. a p. I84). 
platonico delle idee assolute e trascendenti: «Era un sogno di Platone che le idee delle cose esistessero innanzi a queste, in maniera che queste non potessero esistere altrimenti» ${ }^{13}$. In un altro passo Leopardi sostiene che il giudizio umano «non ha a che fare col discorso astratto e metafisico della bellezza» ${ }^{14}$, la quale non può considerarsi «una forma innata, universale e impressa dalla natura nella mente dell'uomo»" ${ }^{\text {IS }}$. Seguendo il pensiero di John Locke, l'autore riconduce la bellezza all' "esperienza che deriva dalle nostre sensazioni». Il sensismo è il fondamento gnoseologico esperienziale, dimostrazione che tutto è in re, nulla ante rem: "Tutto ci è insegnato dalle sole sensazioni» e «nessuna cognizione o idea ci deriva da un principio anteriore all'esperienza». Dunque «supporre il bello e il buono assoluto è tornare alle idee di Platone, e risuscitare le idee innate dopo averle distrutte» ${ }^{16}$. Ciononostante, una volta rigettato il sistema platonico quale fondamento di un assoluto trascendente e di un sistema metafisico, Leopardi sostiene la possibilità di sostituire all'idea platonica il prodotto della fantasia e dell'immaginazione: "Ma noi che abbiamo rigettato il sogno di Platone conserviamo quello di un tipo immaginario del bello» ${ }^{17}$. Dopo avere combattuto l'innatismo, quindi, Leopardi salva l'idea come illusione, in virtù della necessità e dell'amore umano per l'assoluto ${ }^{\mathrm{I}}$.

E tuttavia Platone, pur avendo inventato un sistema metafisico insostenibile nella sua verità (potendosi considerare "false e insussistenti» le Idee) ${ }^{19}$, è annoverato da Leopardi tra i filosofi «notabili e singolari anche per la facoltà dell'immaginazione e del cuore», che si distinsero "per una vena e per un genio decisamente poetico»:

Fra gli antichi Platone, il più profondo, più vasto, più sublime filosofo di tutti essi antichi che ordì concepire un sistema il quale abbraccia tutta l'esistenza, e rendesse ragione di tutta la natura, fu nel suo stile nelle sue invenzioni ec. così poeta come tutti sanno ${ }^{20}$.

Un analogo rovesciamento del pensiero platonico è proposto da Foscolo, il quale nelle riflessioni che accompagnano la Chioma di Berenice ipotizza che quell' «idea metafisica» che sosteneva il governo platonico non fosse

I3. G. Leopardi, Zibaldone, cit., f. I54, p. I84.

I4. Ivi, ff. I256-I257, pp. 910 sgg.

I5. Ibid.

I6. Ivi, ff. I339-I34I, pp. 970-97I.

I7. Ivi, f. I55, p. I85.

I8. Ivi, f. I7I4, p. II9I. Cfr. A. Tilgher, La filosofia di Leopardi, Bologna, Boni, I979; G. Ficara, Il punto di vista della natura. Saggio su Leopardi, Genova, Il Melangolo, I996; M. A. Rigoni, Il materialista e le idee, in Il pensiero di Leopardi, Milano, Bompiani, I997, pp. 57-58.

19. G. Leopardi, Zibaldone, cit., f. I55, p. I85.

20. Ivi, f. 3245, p. 2029. 
altro che "una obbliqua satira della specie umana ${ }^{21}$; il poeta dell' Ortis si domanda ironicamente:

E Platone stesso, perché scriveva ad uomini greci, e non agli angioli della sua repubblica, non è forse per l'altezza de' concetti, e per la pittura de' personaggi, e per la passione delle sue narrazioni, e per quell'intrinseco incantesimo del suo stile più poeta d'ogni altro scrittore, e più che non si conviene forse a filosofo? ${ }^{22}$

È dunque nello svuotamento dell'idea come verità e trascendenza che si attua l'acquisizione del platonismo: se tale sistema non è vero, nel senso che l'idea non è, come l'esperienza empirica dimostra, portatrice di quella verità assoluta che ne fa modello trascendente e assiologico, allora il pensiero del filosofo viene rivalutato come sublime illusione, «un sistema fondato sul brillante e sul fantastico» ${ }^{23}$, tentativo di costruire, con l'immaginazione, un sogno che giustifichi l'essere dell'uomo e delle cose nel mondo e lo riconcili con quella trascendenza che gli sfugge.

Questa teoria dell'immaginazione trova consonanza nella poesia europea romantica coeva a Leopardi e a Foscolo, ad esempio in Keats ${ }^{24}$, ma prima ancora nella distinzione tra imagination e fancy elaborata, seppure con alcune differenze, da Coleridge nella Biographia Literaria e da Wordsworth nella Nota a The Thorn del I80o e nelle due Prefazioni alle Lyrical Ballads del $\mathrm{I} 802$ e del $\mathrm{I} 8 \mathrm{I} 5^{25}$. Nella Nota a The Thorn, il poeta sottolinea il collegamento tra pensiero e parola nella tensione creativa che fa delle parole non soltanto "symbols of the passions», ma direttamente cose, "things", attive ed efficaci, che sono esse stesse componenti della passione («active and efficient, which are themselves part of the passion») ${ }^{26}$. Nella Prefazione del I802, il poeta inglese celebra le immagini («pictures») prodotte dall'immaginazione poetica, prendendo però le distanze da coloro che ne inficiano la santità e la verità («the sanctity and truth») con ornamenti transitori e accidentali («by transitory and accidental ornaments», I, vv. 47I-476 ${ }^{27}$. Wordsworth insiste sul potere evocativo dell'immaginazione, capace di andare al di là dei sensi e dell'imitazione

2I. EN VI, p. 308.

22. Ivi, p. 309.

23. G. Leopardi, Zibaldone, cit., f. 352, pp. 330-331.

24. Cfr. R. Portale (a cura di), Omaggio a Keats e Leopardi: atti del simposio internazionale in occasione del bicentenario della nascita di John Keats, Pisa, Università di Macerata, 1997.

25. Si veda G. N. G. Orsini, Coleridge and German Idealism: A Study in the History of Philosophy with Unpublished Materials from Coleridge's Manuscripts, Carbondale, Southern Illinois University Press, I969.

26. S. Gill (a cura di), William Wordsworth, Oxford-New York, Oxford University Press, 1984, p. 594.

27. W. Wordsworth, The Prose Works of William Wordsworth, a cura di W. J. B. Owen e J. W. Smyser, Oxford-New York, Oxford University Press, 1974. 
(«the conferring, the abstracting, and the modifying power of the imagination», III, 266). Mentre la fantasia collega, spesso in maniera arbitraria e capricciosa, mente e natura, l'immaginazione ha la funzione di cogliere l'eterno («to incite and to support the eternal», III, 390) ${ }^{28}$.

Il rapporto tra passioni e immaginazione, e tra immaginazione e verità, è inoltre al centro della poetica di Keats che, nella lettera a Benjamin Bailey datata 22 novembre 1817 , scrive:

I am certain of nothing but of the holiness of the Heart's affections and the truth of Imagination. What the imagination seizes as Beauty must be truth-whether it existed before or not-for I have the same idea of all our passions as of love: they are all, in their sublime, creative of essential beauty. [...] The imagination may be compared to Adam's dream, - he awoke and found it truth ${ }^{29}$.

Intorno a questo principio si costruisce la tensione, compositiva e metaletteraria, dell'Ode on a Grecian Urn (I819), che si conclude con la nota equivalenza tra bellezza e verità: «Beauty is truth, truth beauty,- - that is all / Ye know on earth, and all ye need to know» (vv. 49-50) ${ }^{30}$. L'immaginazione porta alla coincidenza tra bellezza e verità, dove però alla verità corrisponde non l'idea trascendente come in Platone, ma il sogno, la poesia. Ed essa necessariamente soggiace al mistero e all'irrazionale, secondo quella concezione di negative capability riferita da Keats a Shakespeare: "I mean Negative Capability, that is, when a man is capable of being in uncertainties, Mysteries, doubts, without any irritable reaching after fact and reason» ${ }^{31}$. Nell'Ode il poeta invoca la narratrice silvana («Sylvain historian», v. 3) affinché trasformi in canto le raffigurazioni vascolari, per poi celebrare la dolcezza delle non suonate melodie ( Heard melodies are sweet, but those unheard / Are sweeter", vv. II-I2). La dinamica tra lo schiudersi espressivo del referente mitico e la resistenza a questa stessa espressione continua nel corso del carme, animando la "wild ecstasy» (v. Io) che chiude la prima stanza ${ }^{32}$.

Nel saggio Wordsworth and Hölderlin, Paul de Man mette a confronto i due poeti per l'apertura al divino come categoria concettuale e poetica,

28. Cfr. G. Ferreccio, Iconoclastia romantica, in D. Borgogni e R. Camerlingo (a cura di), Le scritture e le riscritture. Discorso religioso e discorso letterario in Europa nella prima età moderna, Perugia, Edizioni Scientifiche Italiane, 2005, pp. 247-280.

29. J. Keats, Selected Letters, Oxford, Oxford University Press, 2002, p. 36.

30. J. Keats, The Complete Poetical Works, Cambridge, Cambridge University Press, 2008.

3I. J. Keats, Selected Letters, cit., pp. 4I-42. Cfr. W. J. Bate, Negative Capability. The Intuitive Approach in Keats, repr. New York, Contra Mundum Press, 2012 (1965).

32. J. Keats, The Complete Poetical Works, cit. ("Amante audace, mai, mai arriverai a baciare, / Benché tu sia vicino al traguardo - ma non ti crucciare; / Lei non può svanire, e tuttavia tu non potrai conquistare quella gioia, / Per sempre amerai, e per sempre lei sarà splendida»). 
percorso mentale e immaginativo che si collega alla storia e al linguaggio ${ }^{33}$. In Hölderlin (ad esempio in Mnemosyne), tuttavia, il contatto con il divino segnava più insistentemente il trapasso dall'immaginazione alla perdita del linguaggio, allo «scacco» del significato o alla sua elusività ${ }^{34}$. L'immaginazione segue una traccia forte, luminosa, ma al tempo stesso vive di un miraggio, di uno spazio lasciato vuoto da quella traccia.

Il mito classico costituisce spesso l'orizzonte di questo miraggio, in una corrispondenza con la natura chiamata a rinnovarsi e a rinnovare. Se Keats invoca la narratrice silvana per fare rivivere l'urna, e se nella canzone di Leopardi Alla Primavera, o delle favole antiche (I822), l'apostrofe alla natura («Vivi tu, vivi, o santa / natura?», vv. 2O-2I) rinnova, nella speranza di quella stessa vita, l'evocazione di ninfe, di «arcane danze» (v. 25) e «sonar d'agresti Pani» (v. 32), nella poesia di Hölderlin Der Arcipelagus (L'arcipelago, i8oo) le domande del poeta alla Grecia, all'antico («der Alter») sono da intendersi come ipotesi di esistenza di un passato che promette di riaffacciarsi al presente come portatore di nuove immagini di bellezza:

Kehren die Kraniche wieder zu dir? und suchen zu deinen

Ufern wieder die Schiffe den Lauf? umatmen erwünschte

Lüfte dir die beruhigte Flut? und sonnet der Delphin,

Aus der Tiefe gelockt, am neuen Lichte den Rücken?

Blüht Ionien? Ist es die Zeit? denn immer im Frühling,

Wenn den Lebenden sich das Herz erneut und die erste

Liebe den Menschen erwacht und goldner Zeiten Erinnerung,

Komm ich zu dir und grüß in deiner Stille dich, Alter!

Immer, Gewaltiger! lebst du noch [...]

Göttlicher! du, du dauertest aus, denn über den dunkeln

Tiefen ist manches schon dir auf und untergegangen ${ }^{35}$.

L'invocazione al possente e all'eterno («Immer, Gewaltiger!») offre una certezza di questa domanda alla vita: «tu vivi [...] ancora» («lebst du noch»). La durata del divino è percepita come tale («Göttlicher! du, du dauertest

33. P. De Man, Wordsworth and Hölderlin, in Id., The Rhetoric of Romanticism, New York-Chichester, Columbia University Press, 1984, pp. 47-65.

34. Ivi, p. 59.

35. F. Hölderlin, Der Archipelagus, in Id., Poesie, a cura di G. Vigolo, Milano, Mondadori, I976 (I958), pp. IOO-IO4 («Tornano a te le gru? e cercano di nuovo la rotta / Verso i tuoi lidi le navi? Spirano desiderate / Brezze a te sul flutto pacato e soleggia il delfino / Attratto dal fondo, col dorso della nuova luce?/ Fiorisce la Jonia? È questo il tempo? Ché in primavera / Quando ai viventi rinasce il cuore ed il primo / Amore si desta negli uomini e le epoche d'oro ricordano, / A te vengo e saluto il tuo silenzio, o antico! // Sempre, o possente! Tu vivi $[\ldots]$ / [...] ancora; [...]. / Tu perdurasti sempre, o divino, ché sopra le buie / Profondità molte cose ti sono già sorte e perite». 
aus»), benché l'abisso o lo scatenato fuoco notturno travolga e divori le belle isole dell'arcipelago nelle sue profondità. Non si manifesta però nella staticità, ma nel divenire, nel movimento che domina in questo paesaggio, dove si alternano la solitudine del dio, il cui lamento è ascoltato soltanto dalla rupe, e l'incertezza dell'uomo che cerca Atene, come il nocchiero, nelle Grazie, cerca e invoca Atlantide:

Sage, wo ist Athen? Ist über den Urnen der Meister

Deine Stadt, die geliebteste dir, an heiligen Ufern,

Trauernder Gott! dir ganz in Asche zusammengesunken,

Oder ist noch ein Zeichen von ihr, daß etwa der Schiffer,

Wenn er vorüberkommt, sie nenn und ihrer gedenke? ${ }^{36}$

La dinamica tra divino e umano si chiude, alla fine del carme, con un riferimento storico che allude all'umanità presente senza poesia, che vaga «come nell'Orco, senza dèi» («wie im Orkus, / Ohne Göttliches»). E tuttavia questa desolazione prelude a una nuova invocazione a un immortale, il dio del mare, affinché lo spirito si ridesti tra le onde «come nuotatore» («der Geist, dem Schwimmer gleich [...]») ${ }^{37}$.

Nell'opera di Foscolo, si avverte un'interessante risonanza di questi motivi poetici e delle teorie che si sviluppano in Europa tra classicismo e romanticismo ${ }^{38}$. Nelle Grazie, in particolare, immaginazione e contenuto mitico si intrecciano a costituire l'uno il referente dell'altro, in maniera il più possibile unitaria (almeno così come era stato il proposito, mai pienamente realizzato, del poeta) ${ }^{39}$. Il divario tra uomini e dèi, tra realtà e ideale, che costituisce uno dei motivi della poesia di Hölderlin, è in Foscolo colmato da quella che si può definire l'invenzione' delle Grazie, "divinità intermedie tra il cielo e la terra, dotate della beatitudine e della immortalità degli dèi, ed abitatrici invisibili fra' mortali» ${ }^{40}$.

La riflessione trova i suoi fondamenti nei Discorsi che precedono la traduzione della Chioma di Berenice, dove Foscolo auspica che la poesia

36. F. Hölderlin, Der Archipelagus, in Poesie, cit., pp. I04-IO5 («Dimmi, Atene dov' è? Dei suoi maestri sulle urne / La tua città, la più amata da te, vicino alle rive / O luttuoso iddio, t’è in cenere tutta crollata? / O ancor v'è un segno di lei, che il navigante almeno / Quando vi passi innanzi la nomini e se ne ricordi?»).

37. Ivi, pp. II6-II7 («Ma tu, immortale, se anche l’inno dei Greci non più / Ti celebra come una volta, o dio del mare, risuonami / Dai flutti sovente nell'anima ancora, ché sopra le acque / intrepido lo spirito, come nuotatore, si addestri $[\ldots] »)$.

38. Per queste corrispondenze tra Leopardi, Foscolo e Hölderlin, si veda ancora L. Derla, L’isola, cit., pp. II4-II7.

39. Cfr. M. Palumbo, Il racconto del mito e la fondazione della comunità: "Le Grazie» di Ugo Foscolo, «Italies», VI/2, 2002, pp. 527-542, p. 536 (ora ripreso in Id., Foscolo, Bologna, il Mulino, 2010). R. Dell'Erede, La triade o l'unità, cit.; A. Bruni, Belle vergini. "Le Grazie» tra Canova e Foscolo, Bologna, il Mulino, 2009; E. Selmi, Mito e allegoria nella poetica del Foscolo, "La Rassegna della Letteratura italiana», XCVIII, 1994, pp. 76-95.

40. EN I, p. 949. 
risieda nel cuore della civiltà, costituendo un modo di intendere il mondo e una forma essenziale al vivere associato. Fulcri di questa concezione sono i concetti di 'mirabile' e di 'passionato': la poesia deve "percuotere le menti col meraviglioso, ed il cuore con le passioni». Al 'mirabile' Foscolo attribuisce la capacità di ridestare nell'immaginazione «simolacri non solamente divini, ma simili a quello cose che sono care e necessarie ai mortali», mentre il 'passionato' si riferisce alla capacità della poesia di colpire prima i sensi e poi l'intelletto. L'uno attinge al cielo e l'altro alla società. Nelle Grazie in modo particolare l'allegoria collega i due mondi garantendo ad essi, sul piano dell'arte, unità: «La favola degli antichi trae l'origine dalle cose fisiche e civili che idoleggiate con allegorie formavano la teologia di quelle nazioni»; nel presente "il velo dell'illusione» lascia trasparire «un mondo di belle e care immaginazioni», preservando l'uomo dalla «noia» e dalle «ansietà della vita» ${ }^{4}$.

La forza del referente platonico quale espressione dell'assoluto, pur in un processo di rovesciamento e di sostituzione della trascendenza analogo a quello leopardiano, è premessa fondamentale anche per Foscolo. Nell'orazione Dell'origine e dell'ufficio della letteratura il poeta trasforma le idee platoniche del bello, del vero, del giusto in deità create dalla fantasia e dalla memoria degli «irrequieti mortali» per eludere la morte:

E la fantasia del mortale, irrequieto e credulo alle lusinghe di una felicità ch'ei segue accostandosi di passo in passo al sepolcro, la fantasia, traendo dai secreti della memoria le larve degli oggetti, e rianimandole con le passioni del cuore, abbellisce le cose che si sono ammirate e amate [...] tenta di mirare oltre il velo che ravvolge il creato; e quasi per compensare l'umano genere dei destini, che lo condannano servo perpetuo ai prestigi dell'opinione ed alla clava della forza, crea le deità del bello, del vero, del giusto, e le adora; crea le grazie, e le accarezza; elude le leggi della morte, e la interroga e interpreta il suo freddo silenzio; precorre le ali del tempo e al fuggitivo attimo presente congiunge lo spazio di secoli e secoli ed aspira all'eternità; sdegna la terra, vola oltre le dighe dell'oceano, oltre le fiamme del sole, edifica regioni celesti, e vi colloca l'uomo e gli dice: Tu passeggerai sovra le stelle: così lo illude, e gli fa obliare che la vita fugge affannosa e che le tenebre eterne della morte gli si addensano intorno; e lo illude sempre con l'armonia e con l'incantesimo della parola ${ }^{42}$.

In una lettera a Vincenzo Monti, Foscolo spiega di voler riservare, per l'anno I8I4 e I8I5, «il tempo e la mente agl'Inni Italiani, scritti con la ragione morale e poetica de' Sepolcri», e "Alle Grazie, ove saranno 
idoleggiate tutte le idee metafisiche sul bello» ${ }^{43}$. Precisa, inoltre, in un passo degli Appunti:

[...] è mio intento di rappresentare le idee metafisiche in modo che lasciando in pace l'intelletto de' lettori si presentino in tante immagini alla loro fantasia; dalle quali immagini desumano i sentimenti che sogliono essere ispirati dalla Grazia, ed ispirarla ${ }^{44}$.

Con «idee metafisiche», perciò, il poeta si riferisce non alla sfera trascendente, a cui si accede con l'intelletto, ma alla rappresentazione della fantasia e alla sua traducibilità in linguaggio, e precisamente in un linguaggio il più possibile pittorico, evocativo. Analogamente la poesia, sulla scorta di Vico, diventa atto gnoseologico e speculativo, forma privilegiata di conoscenza ${ }^{45}$. Risulta inoltre fondamentale il legame tra passione e immaginazione, tra - per riprendere le parole sopra citate di Keats nella lettera a Bailey — «Heart's affections» e «the truth of Imagination». Nel Saggio sopra l'amore del Petrarca, pubblicato nel I823, infatti, Foscolo pone «la immaginativa di Platone» a sostegno di quella «ingegnosa teorica dell'Amore [...] che forma la macchina della poesia in Petrarca» ${ }^{46}$.

Riguardo alla difficile identificazione di un preciso genere letterario per le Grazie, spiega l'autore negli Appunti:

[...] il fondo del carme delle Grazie è didattico, e lo stile è tra l'epico e il lirico; per ciò che nel raccontare $[\ldots]$ una serie di avvenimenti l'entusiasmo del poeta li trasforma in altrettante pitture l'una dipendente dall'altra, e formanti un tutto che come nella poesia lirica il lettore può comprendere non tanto nel ricordarsi i fatti narrati, quanto nel rappresentarsi vivamente le immagini, e gli effetti che ne risultano ${ }^{47}$.

Da ciò si comprende come l'aspetto centrale sia la possibilità di comunicare attraverso pitture, immagini, prodotte dall' «entusiasmo» del poeta che diventano qui sostitutive delle idee platoniche, identificandosi con la Bellezza assoluta quale ideale da trasmettere ${ }^{48}$. Nella composizione delle Grazie, infatti, le idee metafisiche si riconducono a una sorta di ontologia dell'arte: le Grazie sono coloro a cui il poeta chiede «l'arcana / Armoniosa

43. Ep. II, pp. 544-545, n. 707 (dic. 1808 ).

44. EN I, p. 952.

45. Tra gli altri, si veda G. Mazzacurati, Retaggi vichiani nella filosofia e nella storiografia del Foscolo, in M. Santoro, Foscolo e la cultura meridionale, Napoli, Società Editrice Napoletana, 1980, pp. 42-64.

46. U. Foscolo, Saggio sopra l'amore del Petrarca, in Saggi sopra il Petrarca (in inglese nell'originale, qui nella traduzione di C. Ugoni in Opere, Torino, Einaudi-Gallimard, I995, pp. 66I-682; pp. 66I-662).

47. EN I, p. 958.

48. Cfr. A. Bruni, Foscolo fiorentino all'ombra di Canova, "Giornale storico della letteratura italiana", 2003, pp. 206-234, in particolare pp. 215-216. 
melodia pittrice / Della vostra beltà» (I, vv. 4-6) ${ }^{49}$, origine, causa del carme che ritrae la loro medesima bellezza e la rende immanente nella pittura, nell'immagine ${ }^{50}$ :

[...] i poeti giovandosi delle antiche tradizioni, che [...] senza concatenazione veruna allegorica né teologica giunsero a noi su le Grazie, possono bensì abbellire con la mitologia delle ministre di Venere i loro versi, ma non rappresentarle in modo che altri senta tutta la loro amabile deità, e le dipinga in modo che i poeti e i pittori possano farle de' quadri, e i più eleganti possano imparare le loro virtù ${ }^{\text {si }}$.

Foscolo ridefinisce così anche la nozione di allegoria, già discussa nel Discorsi introduttivi alla Chioma di Berenice ${ }^{52}$; il poeta supera il dualismo platonico e neoplatonico ricomponendo nell'arte mondo terreno e dimensione trascendente. Sempre negli Appunti, nella sezione intitolata Dell'architettura del Carme, presenta le Grazie attraverso la compresenza dei «tre sistemi poetico, storico, e metafisico"; essi "costituiscono la macchina del carme, che è tutto allegorico». Ancora una volta, quindi, discute le «idee metafisiche» riportandole alle sfere immanenti dell'arte e dell'immaginazione. Le Grazie sono figure allegoriche in quanto «divinità intermedie tra il cielo e la terra", la cui nascita feconda "di amabili immagini la fantasia». Ed è «secondo le idee metafisiche» che la grazia risulta, in questo senso, "una dilicata armonia che spira contemporaneamente spontanea dalla beltà corporale, la bontà del cuore, e la vivacità dell'ingegno congiunte in sommo grado in una sola persona, e armonia che ingentilisce sommamente e consola la vita educando chi è capace di sentirla all'idea divina del bello» ${ }^{53}$. Le «idee» rimandano a quelle «fantasie soprannaturali» di cui il poeta parla nei Discorsi sulla Chioma di Berenice ${ }^{54}$, e che la poesia lirica traduce accedendo con il linguaggio simbolico a quell' «età del mondo chiamata favolosa» 5 . È il linguaggio poetico che ha la capacità di trarre «da tutti i più astratti pensieri allegorie e pitture sensibili»" ${ }^{56} \mathrm{La}$ parola, scrive Foscolo, «deve farti passare dal noto che mostra evidentemente, all'ignoto a cui tende facendolo sospettare» ${ }^{57}$.

49. EN I, p. 785 .

50. Cfr. M. Palumbo, Il racconto, cit., p. 530.

5I. EN I, p. 956.

52. L. Derla, Allegoria, in L'isola, cit., pp. 65-IIo.

53. EN I, pp. 967, 949.

54. EN VI, p. 301.

55. Ibid., p. 292. Cfr. C. Del Vento, Un allievo della Rivoluzione, cit., p. 218.

56. EN VI, p. 303

57. EN I, p. 963. 
L'allegoria prescinde dunque dalla trascendenza e si collega al linguaggio delle passioni ${ }^{58}$, riferendosi principalmente all'immaginazione e alla sua traducibilità simbolica; nella Dissertation, il poeta, riconducendola al gruppo canoviano delle Grazie, la definisce come «personificazione di un'idea astratta che, agendo più rapidamente e più agevolmente sui nostri sensi e sulla nostra fantasia, conquista con più rapidità l'intelletto" 59 . Aggiunge inoltre, a proposito dell'unicità, dell'armonia e della forza evocativa delle tre divinità:

[...] e se quelle antiche allegorie fossero state illustrate da Platone o da Bacone, avremmo avuto un'ulteriore conferma della opinione avanzata da loro - che le allegorie nascano da una naturale tendenza e propensione della mente umana; - che esse costituiscano il prodotto più piacevole della fantasia; e che la loro applicazione morale sia dettata da una sapienza desiderosa del progresso e della perfezione della vita sociale ${ }^{60}$.

L'allegoria può perciò essere considerata come 'visualizzazione' dell'idea, forma artistica che si traduce in linguaggio: per questo la poesia lirica ha per Foscolo funzione non tanto mnemonica quanto evocativa; rinnovando e superando, attraverso il concetto della «armoniosa melodia pittrice», il concetto oraziano dell'ut pictura poësis, il poeta deve sapere «rappresentarsi vividamente le immagini, e gli affetti che ne risultano" ${ }^{6}$.

Influenzato, nella teoria del mito e del simbolo, da Georg Zoëga e da Christian Gottlob Heyne ${ }^{62}$, Foscolo assegna all'allegoria una valenza 'moderna' e più ampiamente polisemica ${ }^{63}$ che si sviluppa sul piano linguistico-retorico, in analogia con le osservazioni che Paul de Man, in Retorica della temporalità e in Allegorie della lettura, propone di Wordsworth e Coleridge, di Rousseau e di Rilke; in questi autori l'allegoria rimanda a una parola in perenne tensione tra immaginazione e realtà, tra lettera e figura, cecità e visione, tra la promessa di disvelamento di una totalità o di un'origine e la sua frantumazione ${ }^{64}$.

58. C. Del Vento, Un allievo della Rivoluzione, cit., p. 223.

59. EN I, p. I097.

6о. Ibid., pp. IIоO-IIоI.

6I. M. Palumbo, Ugo Foscolo, cit., p. I03.

62. C. Del Vento, Un allievo della Rivoluzione, cit., pp. 216-217.

63. Ibid., p. 217. Foscolo stesso scrive, nella Dissertation, che l'allegoria del Velo «racchiude un significato molto più recondito e complesso" (EN I, p. III5).

64. Se la metafora può essere intesa come «linguaggio del desiderio», il «fallimento della figurazione appare come la distruzione dell'unità che essa prevedeva di stabilire tra la funzione semantica e la struttura formale del linguaggio» (P. De Man, Allegorie della lettura, Torino, Einaudi, 1997 [1979], p. 6I). 


\section{L'Atlantide nelle Grazie}

Consapevole di procedere per un cammino sempre più impervio nella continuazione del carme (pari a quello dantesco di avvicinamento ad Deum, ma di segno diverso), nel III Inno delle Grazie Foscolo si rivolge ai «Sacri poeti» (III, v. 20) affinché illuminino i «solitari campi / ove l'errante Fantasia mi porta / a discernere il vero» (III, vv. 27-29). Ci troviamo, quindi, ancora una volta di fronte all'apparente paradosso secondo il quale alla verità (e alla bellezza) si accede non tanto per via razionale, ma intuitiva, con la Fantasia. La citazione dell'orfismo ( $\mathrm{i}$ «versi arcani / di Anfione», III, vv. 6-7), di Pindaro e Catullo, e dello stesso Orfeo (III, v. 52) costituisce il referente mitico del potere sacro della poesia e della musica intesa come ultimo tramite tra umano e divino. La musica, infatti, come l'arte, rappresenta la possibilità di attingere al segreto del dio, ma si arresta laddove la storia, con nuove violenze e stragi, sovrasta le opere delle Grazie (III, vv. 32 sgg.). Il "paese ideale» risulta infatti segnato da un rapporto più controverso con il divino, in una più accentuata tensione tra mito e Storia, indicata dall'invocazione alla musa Clio (III, v. 30) ${ }^{65}$. È inoltre Amore, raffigurato come simbolo di una funesta e bellicosa, lucreziana ${ }^{66}$, passione sensuale, a mettere in fuga le Grazie, pur suggerendo immagini che mitigano, in modo ingannevole, l'effetto provocato.

Atlantide è allora, in questo paesaggio, dono e castigo: è l' «alta / reggia» che Minerva ebbe «cara», «al par d'Atene» ma tuttora «in pregio», «or quando i Fati non lasciano ad Atene altro che il nome» (III, vv. 56-59). La funzione indispensabile, e tale proprio in quanto allegorica, delle Grazie consiste anche nella mediazione tra due colpe, sottomesse all'ira di Pallade: quella di Tiresia, che rappresenta il contatto diretto ed empio con il dio, e viene punito per avere visto la nudità e la bellezza abbacinante di Minerva mentre si bagnava, secondo la tradizione che Foscolo attinge dai Lavacri di Pallade di Callimaco ("Ahi! Senza pianto / l'uomo non vede la beltà celeste», III, vv. $83-84)^{67}$; e quella degli uomini, divenuti "pigri all'arti e sconoscenti a Giove» (III, vv. 97-98).

65. EN I, pp. 7I5-716.

66. Il riferimento è al De rerum natura, IV, vv. I037-I287. Cfr. U. Foscolo, Letture di Lucrezio, a cura di F. Longoni, Milano, Guerini e Associati, I990.

67. Orlando riporta i frammenti che Foscolo dedica al mito di Tiresia nella Parte seconda del Sommario III relativo all'inno a Pallade e nei versi inseriti nel commento alla Chioma di Berenice (I803). Cfr. U. Foscolo, Le Grazie, cit., pp. 156-I60. 
Nelle Grazie l'isola appare come un miraggio:

Isola è in mezzo all'oceàn, là dove

sorge più curvo agli astri; immensa terra,

come è grido vetusto, un dì beata

d'eterne messi e di mortali altrice

(III, vv. 85-88).

Pur mantenendo la suggestiva collocazione nell'Oceano (cfr. Pl., Criti., II 5b), che segue gli sviluppi del mito dall'età ellenistica fino al Settecento spesso in rapporto ai luoghi delle isole felici ${ }^{68}$, e il ricordo della sua grandezza («immensa terra, / come è grido vetusto», III, vv. 86-87; cfr. Pl., Criti., I08e), nell' «oggi» Atlantide esiste per gli uomini nelle sole voci del desiderio, come nell'illusione del nocchiero che la invoca:

Invan la chiede all'onde oggi il nocchiero, or i nostri invocando or dell'avverso polo gli astri; e se illuso è dal desio, mira albeggiar i suoi monti da lunge, E affretta i venti, e per l'antico grido Atlantide l'appella. [...]

(III, vv. 89-94).

Come all'Atene di Hölderlin, così all'Atlantide di Foscolo si chiede di riemergere attraverso l'atto di evocazione poetica e di nominazione intesa come appropriazione (seppure impossibile), come blochiano principio speranza, antidoto contro la Storia, spiegazione e riparo. In una variante, il nocchiero «illuso / Biancheggiar mira i suoi monti da lunge»" dove «biancheggiare» rimanda alla Teresa dell'Ortis e alla figura della «sacra danzatrice» dell'Inno a Vesta. In quest'ultimo il movimento si presta al prodursi dell'immagine («Ma se danza, / vedila!», II, vv. 30-3I); e la poesia, intesa come pittura, delinea la figura della danzatrice trovando forza evocativa nella sottrazione di essa quasi per dissolvenza ( $\mathrm{E}$ chi pinger la può? [...] Mentre a ritrarla / Pongo industre lo sguardo, ecco m'elude [...] appena veggio / Il vel fuggente biancheggiar fra' mirti», vv. 35-44 passim $)^{70}$.

Se Atlantide esiste, però, è in quanto "dono» per le Grazie - e, indirettamente, per gli uomini - come dono è il Velo, che viene intessuto, proprio in quel paese ideale, ad opera delle dee radunate da Pallade per raffigurare (attraverso la poesia come atto speculativo, ermeneutico) la vita, gli affetti, le passioni. L'isola garantisce quindi l'unico possibile accesso

68. Cfr. M. Ciardi, Atlantide, Roma, Carocci, 2002.

69. EN I, pp. 829 e sgg.

70. EN I, p. 744 . 
all'età aurea nel presente: il culto per le «idee metafisiche» come immagini della mente e del cuore, e per le Grazie concepite come allegorie, forme di rappresentazione dell'ideale di bellezza assoluta e, in quanto "divinità intermedie tra il cielo e la terra", tramite di una visione pura, molteplice e indiretta del divino (e non diretta e accecante, come quella, colpevole, di Tersite), che prova a tradursi in linguaggio.

Atlantide è, in questo senso, utopia di perfetta coincidenza tra estetica ed etica, mito di un paesaggio che associa alla bellezza la funzione civilizzatrice:

Onde, qualvolta per desìo di stragi si fan guerra i mortali, e alla divina libertà danno impuri ostie di sangue; o danno a prezzo anima e brandi all'ire di tiranni stranieri, o a fera impresa seguon avido re che ad innocenti popoli appresta ceppi e lutto a' suoi; allor concede le Gorgòni a Marte Pallade, e sola tien l'asta paterna con che i regi precorre alla difesa delle leggi e dell'are, e per cui splende a' magnanimi eroi sacro il trionfo. Poi nell' isola sua fugge Minerva, e tutte Dee minori, a cui diè Giove d'esserle care alunne, a ogni gentile studio ammaestra: e quivi casti i balli, quivi son puri i canti, e senza brina i fiori e verdi i prati aureo il giorno sempre, e stellate e limpide le notti (III, vv. IOI-II9).

Negli ultimi versi è evidente, appunto, la riscrittura foscoliana dell'età dell'oro che trasforma la matrice esiodea e platonica, e consiste nella possibilità di immaginare — per tramite di Minerva e delle Grazie la possibilità di uno spazio, dell'isola oltre la Storia. Se infatti nei miti politici di Platone l'approssimazione al divino (e il recupero simbolico dell'età dell'oro) avveniva con la comprensione intellettuale, memoriale e razionale, dell'idea e preludeva alla sua applicazione in un contesto storico, in Foscolo il divino si manifesta nella poesia, con la corrispondenza tra verità, immaginazione e bellezza, e diventa parte di un progetto etico ed educativo totalizzante ${ }^{71}$. 
Ha scritto Leopardi riferendosi a Rousseau: «Le pays de chimères est en ce monde le seul digne d'être habité et tel est le néant des choses humaines, que hors l'être existant par lui-même, il n'y a rien de beau que ce qui n'est pas» ${ }^{72}$. Nella poesia successiva, tuttavia, Atlantide tenderà a confondersi con meno nitidi pays de chimères: in Le Voyage di Baudelaire saranno gli amari miraggi, i nudi isolotti dalla fisionomia di Eldoradi a nutrire le illusioni del vecchio accattone, sognatore di «brillants paradis», confondendo follia e immaginazione e degradando quest' ultima rispetto alla precedente funzione conoscitiva ("L'Imagination qui dresse son orgie / ne trouve qu'un récif aux clartés du matin», II, vv. I5-I6). 